\title{
Subgrid particle resolution for the turbulent transport of a passive scalar
}

\author{
G.-H. Cottet, G. Balarac and M. Coquerelle \\ CNRS and Université de Grenoble, 38041 Grenoble Cedex 9, France \\ Georges-Henri.Cottet@imag.fr
}

\section{Introduction}

The transport of passive scalar in turbulent flows remains a challenging and important problem for several reasons. First, the passive scalar spectra exhibit various behaviors depending on the molecular Schmidt number value (see [3]). The passive scalar spectrum with a high Schmidt number exhibits thus a $k^{-1}$ scaling between the Kolmogorov scale and the Batchelor scale [1]. This suggests that it is more demanding in terms of spatial resolution than the momentum. Moreover, capturing accurately advected scalar quantities is of crucial important in reacting flows, as concentrations controls reaction rates [5]. In Large Eddy Simulations, classical subgrid-scale models, which only focus on dissipation related to the transfer of energy to small scales, are not sufficient to predict adequately the effect of non-resolved small scales of the advected concentrations on the large scales involved in the reactions. Another field where passive scalar requires special care is the case of free surface multiphase flows when interfaces and surface tensions are captured by a level set method. The idea of using a different resolution for the scalar ad the momentum is therefore natural. This paper investigates the use of a subgrid particle methods to predict accurately small scales while keeping the computational cost at a reasonable level.

\section{The numerical method}

We consider the incompressible Navier-Stokes equation, written in the vorticity formulation, coupled with and advection equation for a passive scalar:

$$
\begin{aligned}
\boldsymbol{\omega}_{t}+(\mathbf{u} \cdot \boldsymbol{\nabla}) \boldsymbol{\omega}-(\boldsymbol{\omega} \cdot \boldsymbol{\nabla}) \mathbf{u}-R e^{-1} \Delta \boldsymbol{\omega} & =0 \\
\phi_{t}+(\mathbf{u} \cdot \boldsymbol{\nabla}) \phi & =0 .
\end{aligned}
$$

This system is soved by a remeshed particle method [2]. This method can be summarized as follows: particles are initialized in the support of the initial 
vorticity and scalar fields. At each time-step they are advected by the local velocity field then remeshed on a regular fixed using high order interpolation formulas. On that grid a classical diffusion finite-difference formula is used to update vorticity values and velocity is deduced by FFT-based fast solvers. To advect particles in the next step, velocity values are interpolated on particle locations using the same formula as in the remeshing step. A validation of the method against spectral method in DNS of homogeneous isotropic turbulence can be found in [2]. The method exhibits two features which are particularly important in the context of the advection of passive scalar. Particles remain in the support of the advected quantities and the method is not constrained to usual CFL stability conditions. This means that if particles are used at a higher resolution for the simulation of the scalar than for the vorticity, it is not necessary to use smaller time-steps to update the scalar field. If in addition the scalar is concentrated on small parts of the computational domain, subgrid particle resolution can be used at a marginal additional cost.

\section{Results}

We first consider a jet at a low Reynolds number carrying a passive scalar. The Schmidt number is taken infinite. We compare two experiments. In the first one, both vorticity and scalar are under-resolved. In the second one, the vorticity is under-resolved but the scalar use a three times finer resolution. Figure 1 is a volume rendering visualization of the density for both cases. Although the second experiment takes only $20 \%$ more CPU time than the first one - due to the spatial localization of the scalar - there is a clear gain, at least from a qualitative point of view, in the visualization of the intermediate scales.
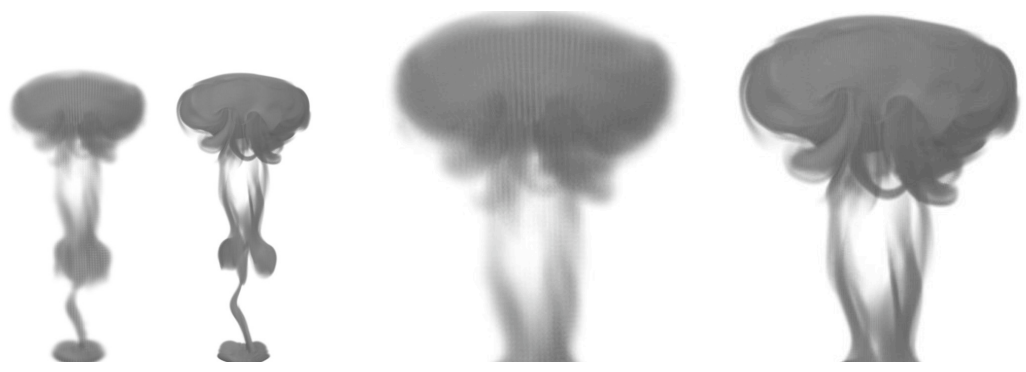

Fig. 1. Left pictures: volume rendering comparison of a low (left pictures) and high (right pictures) resolution of scalar in an under-resolved jet. The right pictures are a close-up view of the top part of the jet.

We next give more quantitative illustrations on a classical turbulence experiment. The flow and scalar are initialized by turbulent fields in a periodic 
box, with a spectral peak at a low wave number. The initial Reynolds number based on the Taylor micro-scale is 48.4 corresponding to a viscosity $\nu=0.01$. The Schmidt number is chosen equal to 10 . We compare the results obtained on the one hand by a full DNS using $N=128$ grid points in each direction for both the flow and the scalar, to the results given on the other hand by a simulation combining a LES of the flow with $N=32$ with a DNS of the scalar using $N=128$ points (we will refer to this later simulation as an hybrid $L E S-D N S$ ). In order to be able to compare side by side scalar visualizations, we have used in the second case the velocity field obtained by filtering the full DNS field, with a top-hat filter in the Fourier space. We show in figure 2 the spectra for the scalar at two given times $(t=0.6$ and $t=1.4)$. One may observe on the spectra that the hybrid LES-DNS gives a satisfactory scalar spectrum all the way to the smallest scale. It can be noticed that the spectrum shows a plateau that extends a $k^{-1}$ scaling further than in the full DNS. This $k^{-1}$ scaling is not the Batchelors scaling but this spectral behavior has already been found by Lesieur and Rogallo [4]. They explain this spectral behavior as a result of the shearing by large-scale velocity gradients. Whether in the present case this is a desirable behavior will be seen by comparing these spectra with DNS using $N=256$ grid points for the scalar.

Figure 3 gives a visualization of the surfaces for the same scalar value 0.9 together with the contours of the scalar in a plane through the middle of the computational box. This comparison shows that the hybrid LES-DNS captures
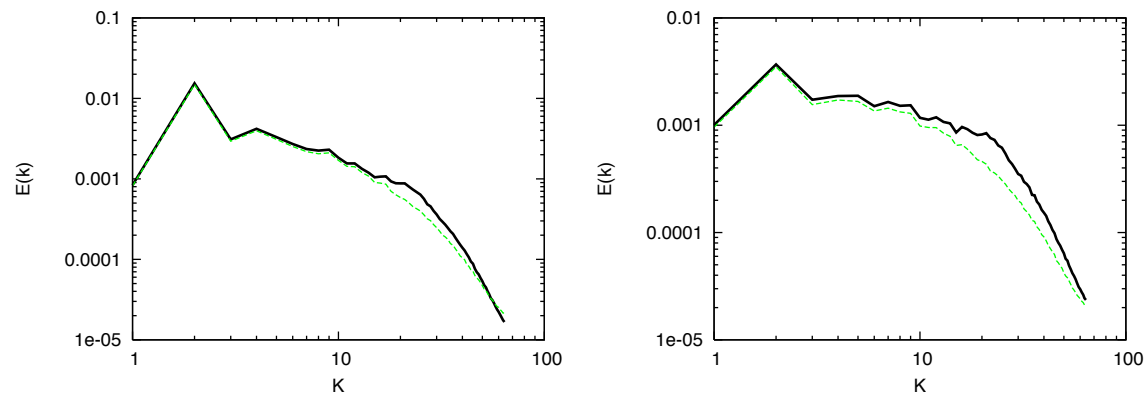

Fig. 2. Scalar spectra in an homogeneous turbulence experiment by a full $128^{3}$ DNS (green dotted line), and a $128^{3}$ scalar DNS in a filtered flow with $N=32$ (solid black line) at times $t=0.6$ (left picture) and $t=1.4$ (right picture).

all the visible scales obtained in the full DNS. Some additional small scale details are also obtained in parts of the flow. This observation is confirmed by looking at the filtered scalar fields corresponding to these two simulations (bottom pictures of Figure 3). Whether these additional features are desirable or numerical artifact is the subject of ongoing higher resolution simulations. 

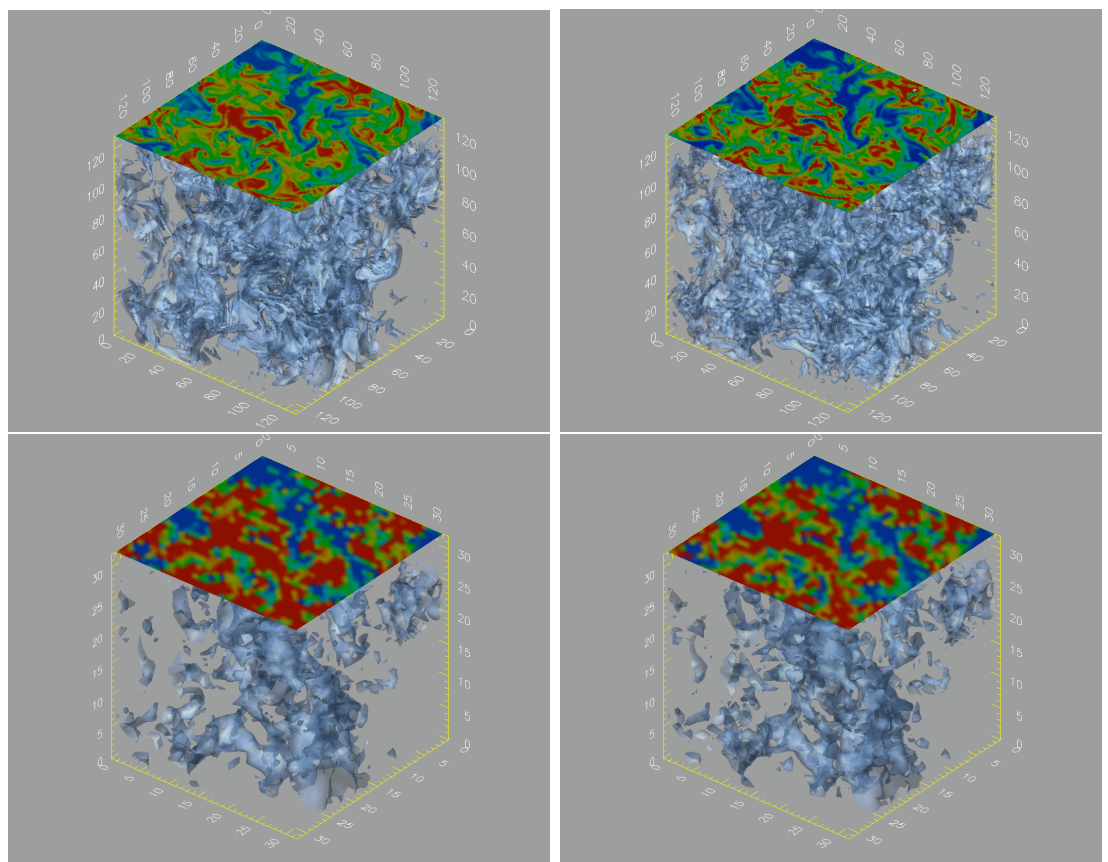

Fig. 3. Isosurface corresponding to $\phi=0.9$ and contours through the middle of the computational box for the scalar in a full DNS (top-let picture) and in an hybrid LES-DNS (top-right picture). Bottom pictures: scalar fields filtered at $k=1 / 32$.

\section{Conclusion}

We have described a numerical technique based on particle methods for the turbulent transport of scalar combining LES models for the momentum and DNS for the scalar. Numerical validations on homogeneous turbulent flows indicate that this method has the ability to reproduce fine scales for the scalar with a minimal computational effort and can thus be viewed as an LES tool for the scalar transport. Ongoing works concern a systematic use of this approach to explore spectrum decay as a function of the Schmidt number and its application to interface capturing in multiphase flows.

\section{References}

1. G.K. Batchelor. J. Fluid Mech., 5, 1959.

2. G.-H. Cottet, B. Michaux, S.Ossia and G. Vanderlinden, J. Comp. Phys, 175, 2002 .

3. M. Lesieur, Turbulence in Fluids 4th Edition, Springer, 2008.

4. M. Lesieur and R. Rogallo, Phys. Fluids A 1, 718, 1989.

5. H. Pitsch, Annu. Rev. Fluid Mech. 38, 453, 2006. 